\title{
Applied Research of NMR Movable Fluid Saturation in Development of Low Permeability Oilfield
}

\author{
Gao Xingjun ${ }^{1}$ Cao Baoge ${ }^{2 *}$ li Yuanliu ${ }^{1}$ and Xiao Ling $^{2}$ \\ ${ }^{1}$ Hengshan Oil Production Plant, Yanchang Oilfield, Yulin, Shanxi, 719100, China \\ ${ }^{2}$ Petroleum Engineering Institute, Xi'an Shiyou University, Xi'an, Shanxi, 710065, China
}

\begin{abstract}
In order to improve the production degree of reserves in low permeability oilfield, taking Chang 2 reservoir in Bailangcheng oil region as an example, the movable fluid saturation and its influencing factors were studied by using NMR and high speed centrifugal method, on this basis, the application of movable fluid saturation in low permeability oilfield development is studied. The results show that the pore radius of low permeability reservoir is large, the micro-fine throat is developed, the movable fluid mainly exists in the volume controlled by the throat, the saturation of the movable fluid is low, and the production is difficult. Based on the relationship between the movable fluid saturation and permeability, single well geological reserves and permeability, availability of reserves and remaining movable reserves are evaluated, and the key areas of oilfield development adjustment are determined. According to the movable fluid saturation, the reservoirs are classified to predict the theoretical development effect of the reservoir, and the actual development effect of the oilfield is evaluated by the ratio of the recoverable reserves and movable reserves predicted by the dynamic method, the adjustment space of oil field is analysed. The results of this paper have important reference value for the development policy and adjustment policy of low permeability reservoir.
\end{abstract}

\section{Introduction}

The proportion of low permeability reservoir reserves in proved unused reserves in China is as high as 60\% above[1], but the real and valuable reserves are movable resources[2-3]. The movable fluid saturation can be used to evaluate the oil and gas availability directly and quickly. The most commonly used method to study the saturation of movable fluid is the one of NMR and high speed centrifugation, which has been widely used to study the characteristics of movable fluid and microscopic pore structure characteristics in low permeability and tight oil and gas reservoirs in recent years[4-9], the movable fluid saturation can also be used to classify and evaluate the reservoir [10-13]. At present, there are few studies on the combination of movable fluid parameters and oilfield development effect[14-16], and these researches only stay at the stage of qualitative analysis, there are few studies on the evaluation of oil and gas reserves, development effect and adjustment potential of oil field by using mobile fluid. Due to the poor physical properties of low permeability reservoir, a large part of the fluid in the reservoir can not flow because of the capillary force and adhesive force, and the deliverability of the reserves is low. In this paper, taking Chang 2 reservoir in Bailangcheng oil region as the research object, based on the analysis of the movable fluid saturation and its influencing factors, the application of the movable fluid saturation in oilfield development is analysed in combination with the actual development situation.

\section{Experimental Study of movable fluid saturation}

The cores are drilled from rock pillar of Chang 2 reservoir, then washed off the oil, dried, and $100 \%$ saturated formation water. In accordance with SY / T6490-2007 industry standard, Nuclear magnetic resonance $T_{2}$ spectroscopy of $100 \%$ saturated water cores and cores centrifuged by high speed centrifugal force of $300 \mathrm{psi}$ $(2.07 \mathrm{MPa})$ is measured using the Newmeyer nuclear magnetic resonance apparatus.

\subsection{Characteristics of nuclear magnetic resonance $\mathrm{T}_{2}$ spectrum}

The $T_{2}$ cutoff value is determined using the method of core nuclear magnetic resonance experiment (Fig.1). The $\mathrm{T}_{2}$ spectra of $100 \%$ saturated water and centrifugal to irreducible water are measured respectively, and then the $\mathrm{T}_{2}$ spectra are accumulated from the lower end, the accumulation curve of the saturated spectral area is obtained, and then the accumulation curve of the Centrifugal $T_{2}$ spectrum is obtained from the lower end of the spectrum, and the maximum of the accumulation curve of the centrifugal spectral area is taken as a line perpendicular to the vertical axis, there is a point of 
intersection between the line and the accumulated curve of Saturated Spectral area, and the corresponding value of the intersection on the Saturated $T_{2}$ spectrum is the $T_{2}$ cutoff value[1,14,18]. The percentage of mobile fluid can be obtained by dividing the difference of the $\mathrm{T}_{2}$ accumulation curve of the sample before and after centrifugation by that of the sample before centrifugation[1,14].

According to the experimental results (Table 1 and Fig. 2 ), the $T_{2}$ spectra of saturated simulated formation water are mainly bimodal and trimodal, and the peak values are high on the left side, which indicates that the pore of rock is mainly small pore and the reservoir physical property is poor. Comparing the positions of the $T_{2}$ cutoff values of different cores in the $T_{2}$ spectrum, the movable fluid is distributed in both small and medium and large pores, and the lower the permeability of the Rock, the more the movable fluid is in the small pores, and the lower the saturation of the movable fluid, the harder it is to exploit the fluid. For example, the permeability of Z24-2-6 and $\mathrm{Z} 71-1-2$ cores is $0.522 \times 10^{-3} \mu \mathrm{m}^{2}$ and $0.797 \times 10^{-3} \mu \mathrm{m}^{2}$ respectively, and the movable fluid saturation is also low, only38.01\% and $54.79 \%$, respectively.

Table 1. Experimental results of NMR movable fluid saturation in Chang 2 reservoir

\begin{tabular}{ccccccc}
\hline $\begin{array}{c}\text { Sample } \\
\text { number }\end{array}$ & Porosity/\%/ & Permeability $/ 10^{-3} \mu \mathrm{m}^{2} /$ & $\begin{array}{c}\text { NMR } \\
\text { porosity } / \%\end{array}$ & $\mathrm{~T}_{2 \text { cutoff }} / \mathrm{ms}$ & $\begin{array}{c}\mathrm{BVI} \\
\mathrm{S}_{\mathrm{wi}} / \%\end{array}$ & $\begin{array}{c}\text { Movable fluid } \\
\text { saturation } / \%\end{array}$ \\
\hline Z24-2-3 & 17.59 & 107.3 & 4.1056 & 0.7402 & 28.03 & 71.97 \\
Z24-2-6 & 14.96 & 0.522 & 3.0158 & 0.9116 & 61.99 & 38.01 \\
Z24-2-8 & 16.63 & 33.9 & 4.9514 & 0.9116 & 38.23 & 61.77 \\
Z71-1-2 & 11.43 & 0.797 & 2.7186 & 0.6906 & 45.21 & 54.79 \\
Z71-1-3 & 8.71 & 1.23 & 3.2263 & 1.8252 & 50.65 & 49.35 \\
Z71-1-6 & 8.14 & 10.9 & 1.3374 & 2.7683 & 38.66 & 61.34 \\
Z24-2-7 & 13.56 & 15.3 & 3.2986 & 0.8504 & 40.26 & 59.74 \\
\hline
\end{tabular}

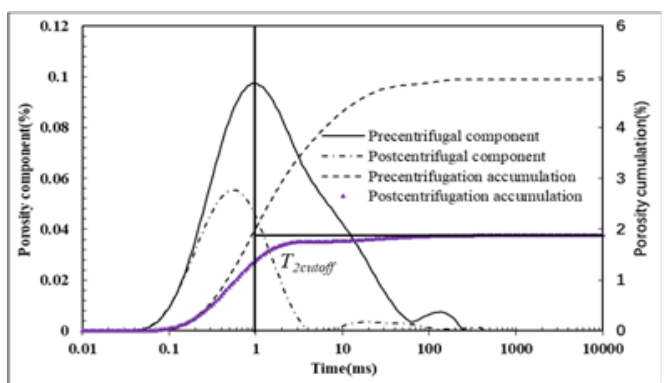

Fig. 1 Determination method of $\mathrm{T}_{2}$ spectrum of Z24-2-8 core in Chang 2 reservoir

\subsection{Influencing factors of mobile fluid saturation}

According to the results of nuclear magnetic resonance experiment, the movable fluid saturation of 7 cores in Chang 2 reservoir is $38.01 \% \sim 71.97 \%$, and the average is $56.71 \%$. Compared with other low permeability reservoir[14], the reservoir has a higher percentage of movable fluid, which shows that the oilfield has a greater development potential. Combined with the results of the constant rate mercury-injection experiment with parallel cores (Fig.3-Fig.9), the main factors affecting the saturation of the movable fluid can be analysed.

(1) Influence of reservoir physical properties

The study shows that there is a strong logarithmic relationship between the movable fluid saturation and the reservoir permeability of sandstone reservoirs above the low permeability level[10-12,19]. Chang 2 reservoir is a typical low permeability reservoir. The correlation between movable fluid saturation and permeability is the strongest among all parameters, and the correlation between movable fluid saturation and porosity is the worst

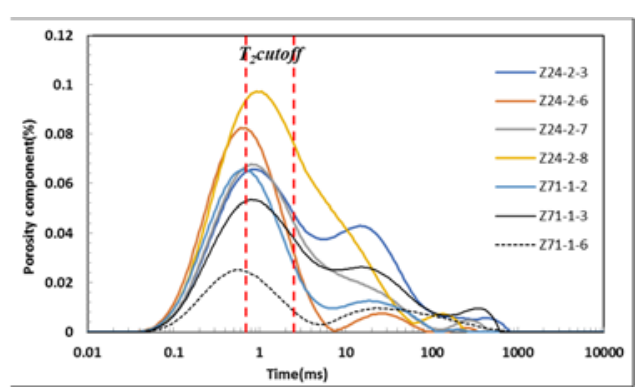

Fig.2 The porosity component of NMR $\mathrm{T}_{2}$ spectra of watersaturated state in Chang 2 reservoir

(Fig.3-Fig.4). Movable fluid saturation is defined as the percentage of pore volume of fluid that can flow under the pressure difference of reservoir development. The concept of movable fluid saturation emphasizes that under the condition of a certain pressure difference, the amount of movable fluid in the reservoir and the fluidity of the fluid is proportional to the permeability when the pore volume is constant, that is, the higher the permeability of the Rock, the more the saturation of movable fluid is. Of course, under the condition of a certain permeability, the saturation of movable fluid depends on the porosity, so the permeability is the main factor that affects the saturation of movable fluid.

(2) The influence of Pore radius, throat radius and pore-throat ratio

Of the three parameters, the pore-throat ratio has the largest effect on the movable fluid saturation and the pore radius has the smallest effect (Fig.5-fig.7). The ratio of Pore to throat indicates the relative size of Pore and throat, which determines the saturation of movable fluid. The Pore of Chang 2 reservoir is mainly medium pore, the 
micro-fine throat is developed and the pore-throat ratio is large[17]. The larger pore-throat radius ratio reflects that the larger pore is surrounded by the smaller throat, and the flow resistance of fluid through the throat is high, so it is easy to be blocked, the fluid saturation is low, and the recoverable degree of oil is low.

(3) The influence of effective pore volume and effective throat volume

The movable fluid saturation has a good positive correlation with the effective throat volume per unit rock sample, and has a certain correlation with the effective pore volume per unit rock sample (Fig. 8-Fig.9). The ratio of effective throat volume to effective pore volume is in the range of $0.67 \sim 2.31$, which shows that the movable fluid mainly exists in the throat-controlled volume. The smaller the throat radius, the more fluid in the throatcontrolled volume, the more difficult the fluid flow, the correlation between mobile fluid saturation and effective throat volume is stronger. There is a positive correlation between the ratio of effective throat volume to effective pore volume and permeability (Fig. 10), which shows that the larger the permeability, the smaller the effective throat volume and the larger the effective pore volume, the easier

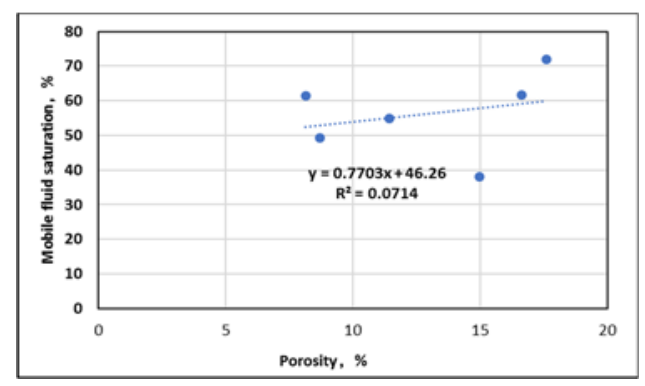

Fig.3 Relationship between movable fluid saturation and porosity

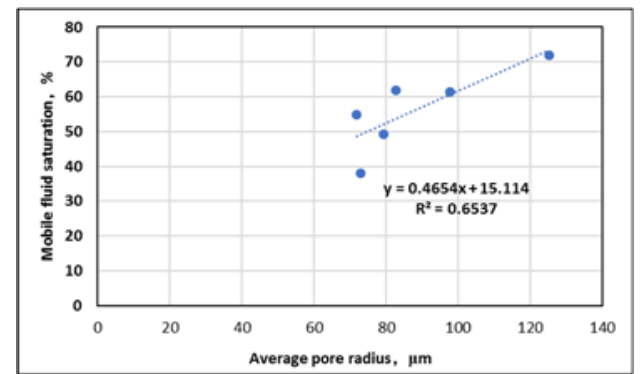

Fig. 5 Relationship between mobile fluid saturation and average pore radius

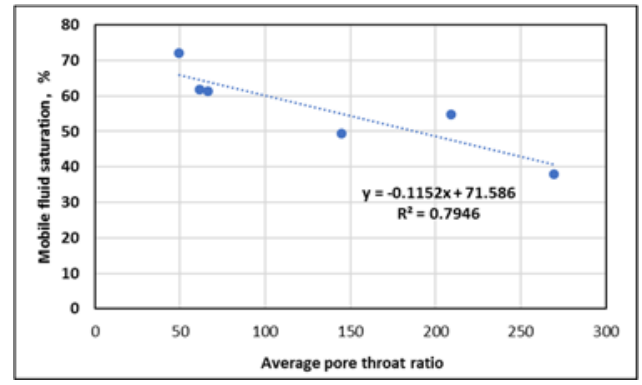

Fig. 7 Relationship between mobile fluid saturation and average pore throat ratio the fluid flow is, the greater the saturation of the movable fluid.

Combining the above studies, the permeability of Chang 2 reservoir is mainly distributed in the range of 1.0 $\sim 20 \times 10^{-3} \mu^{2}$, and the average permeability is $15.1 \times$ $10^{-3} \mu^{2}$. Although the pore radius of reservoir is large, the effective volume controlled by micro-fine throat is obviously larger because of the development of micro-fine throat, and the movable fluid mainly exists in the volume of the effective throat pore, so the fluid to exploit is relatively difficult.

\section{Application of NMR movable fluid saturation in oilfield development}

\subsection{Study the degree of reserves availability and evaluate the development potential of oil field}

Chang 2 reservoir of Bailangcheng oil region has experienced elastic energy recovery and full water injection development from when the industrial oil flow was discovered in drilling well 20 in June 1993.

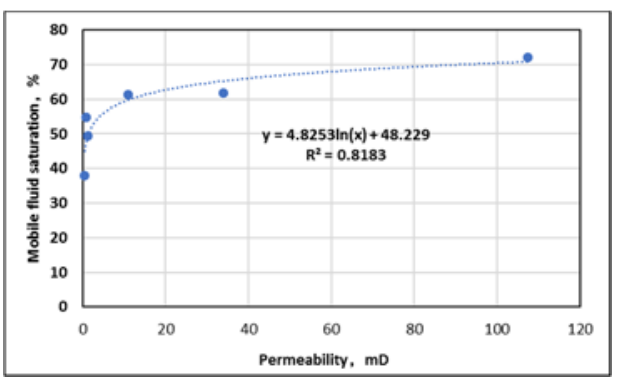

Fig.4 Relationship between movable fluid saturation and permeability

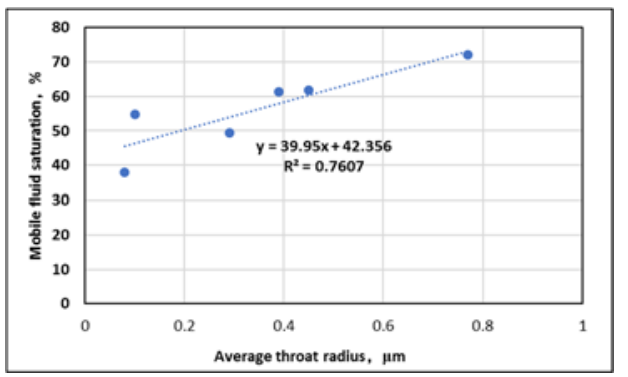

Fig. 6 Relationship between mobile fluid saturation and average throat radius

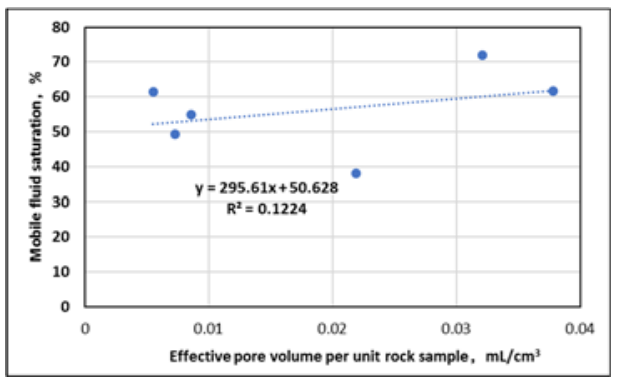

Fig. 8 Relationship between mobile fluid saturation and effective pore volume per unit rock sample 


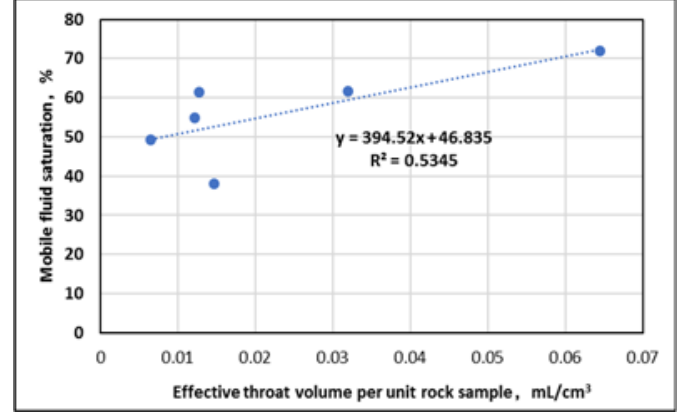

Fig. 9 Relationship between mobile fluid saturation and effective throat volume per unit rock sample

By the end of 2017, the degree of reserve recovery was $19.03 \%$, and the water cut was $73.1 \%$. Because of the development of micro-fine throat and the difference of reservoir physical properties, the recoverable degree of oil and gas is restricted[17], which leads to the great difference of plane producing degree of reserves. In order to improve the degree of reserves production, it is necessary to do a good job in the evaluation of development potential, and make clear the size and distribution of the movable reserves and the remaining movable reserves. Therefore, on the basis of determining the lower limit value of reserve calculation parameters, the oil saturation and porosity of a single well are calculated by using the thickness weighted average method[21-22], and the control area of a single well is calculated by using the triangular network growth algorithm[23], then the controlled reserves of single well are calculated by using the volumetric method, and the movable reserves are calculated according to the relationship between permeability and movable fluid saturation (Table 2 and Fig.11-Fig.12).

The geological reserves of Chang 2 reservoir are $1200.4 \times 10^{4} \mathrm{t}$, the movable reserves are $639.0 \times 10^{4} \mathrm{t}$, and the

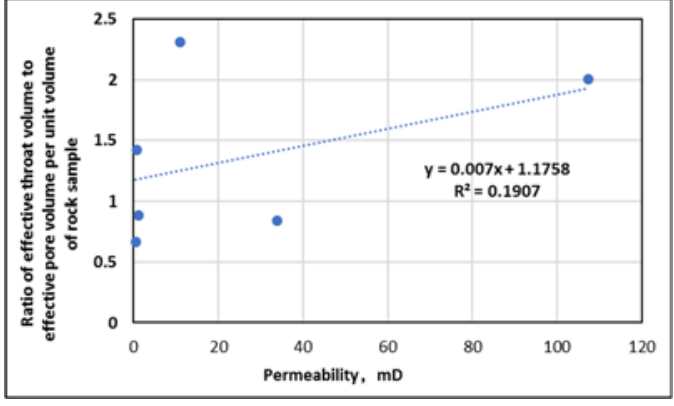

Fig. 10 Relationship between ratio of effective throat volume to effective pore volume per unit volume of rock sample

recoverable degree of the reserves is $53.22 \%$, which shows that the potential of oilfield development is great. According to the numerical value, the single well geological reserves, the movable reserves and the remaining movable reserves can be divided into four categories. The geological reserves of single well are mainly in class I and Class II, mainly in the middle and northeast of the reservoir, and the wells with low geological reserves are mainly in the edge of the reservoir.

The distribution of movable reserves inherits the distribution characteristics of geological reserves, but it is restricted by reservoir permeability. The movable reserves of single well are mainly III reserves, which are distributed in large area in the study area. The movable reserves of Type II are low, mainly located in the north of the fault in the central part of the reservoir, and a small amount in the northeast part of the reservoir. Class IV and class I movable reserves are very low, class IV movable reserves are mainly located in the edge of the reservoir, and Class I movable reserves are scattered in the middle of the reservoir.

Table 2. Summary of reserves calculation for Chang 2 reservoir

\begin{tabular}{|c|c|c|c|c|c|c|c|}
\hline \multicolumn{2}{|c|}{ Single well reserves } & \multicolumn{2}{|c|}{ Geological reserves } & \multicolumn{2}{|c|}{ Movable reserves } & \multicolumn{2}{|c|}{$\begin{array}{l}\text { Remaining movable } \\
\text { reserves }\end{array}$} \\
\hline Categories & Reserves $/ 10^{4} \mathrm{t}$ & Reserves $/ 10^{4} \mathrm{t}$ & $\begin{array}{c}\text { Percentage } \\
\text { of total } \\
\text { reserves } / \%\end{array}$ & Reserves $/ 10^{4} t$ & $\begin{array}{c}\text { Percentage } \\
\text { of total } \\
\text { reserves } / \%\end{array}$ & Reserves $/ 10^{4} \mathrm{t}$ & $\begin{array}{l}\text { Percentage } \\
\text { of total } \\
\text { reserves } / \%\end{array}$ \\
\hline I & $>5.01$ & 523.3 & 43.6 & 34.4 & 5.4 & 10.9 & 2.5 \\
\hline II & $3.01-5$ & 417.8 & 34.8 & 186.8 & 29.2 & 85.0 & 19.1 \\
\hline III & $1.01-3$ & 232.3 & 19.4 & 356.7 & 55.8 & 269.7 & 60.6 \\
\hline IV & $<1$ & 26.9 & 2.2 & 61.1 & 9.6 & 79.5 & 17.9 \\
\hline \multicolumn{2}{|c|}{ Total } & 1200.4 & 100 & 639.0 & 100 & 445.1 & 100 \\
\hline
\end{tabular}




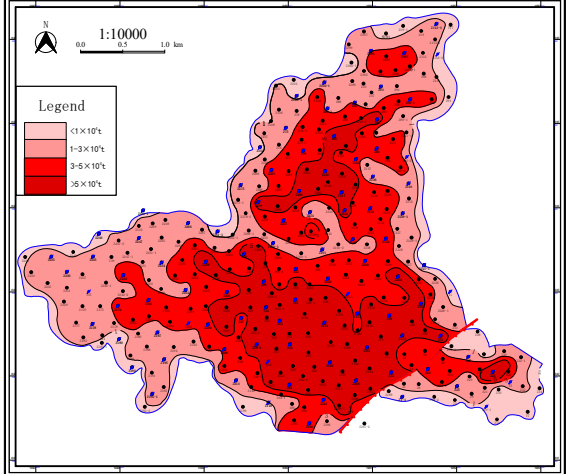

Fig.11 Single well reserve distribution diagram of Chang2 reservoir

\subsection{Determining the key point of oilfield development adjustment according to the size and distribution of remaining movable reserves}

The total remaining movable reserves of a single well is $445.1 \times 10^{4} \mathrm{t}$, accounting for $69.7 \%$ of the movable reserves. The residual movable reserves of single well are mainly Class III reserves, accounting for $60.6 \%$ of the total, which

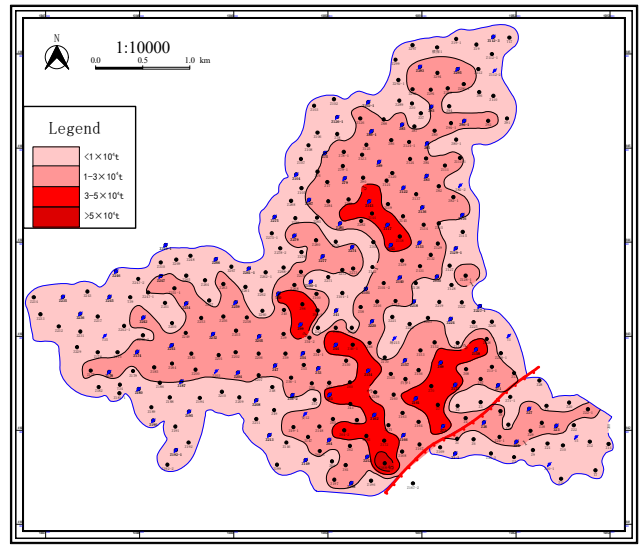

Fig. 13 Distribution map of remaining movable reserves in Chang 2 reservoir

\subsection{Classification of reservoirs}

The movable fluid saturation in the reservoir reflects the recoverable degree of the crude oil, and the reservoir can be classified according to the movable fluid saturation. The recoverable degree of the crude oil or average movable fluid saturation in Chang 2 reservoir is $53.22 \%$. According to the classification standard of movable fluid saturation in table $3[11,24]$, it is generally a better reservoir, and mainly belongs to type II reservoir, and type III reservoir occupies a lower percentage, scattered throughout the reservoir. Theoretically speaking, the development of Chang 2 reservoir can achieve better results.

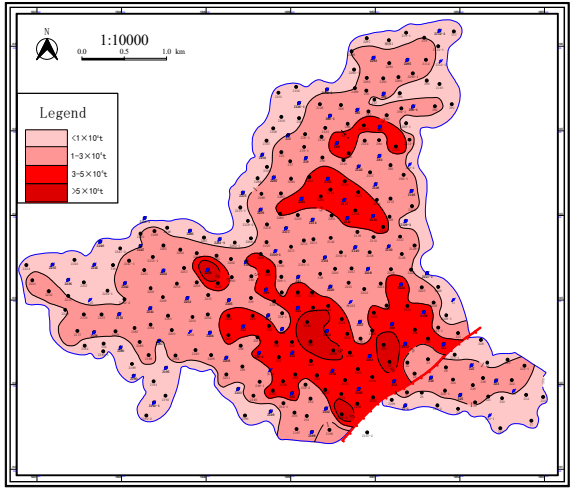

Fig.12 Single well movable reserve distribution diagram of chang2 reservoir

are distributed in a large area in the reservoir. The remaining movable reserves of Class II and Class IV are close to each other. Class II reserves are mainly located between individual well groups north of the central fault in the reservoir(Fig.13 and Table 3). According to the size and distribution of the remaining movable reserves, there is great potential for further development and adjustment of the reservoir.

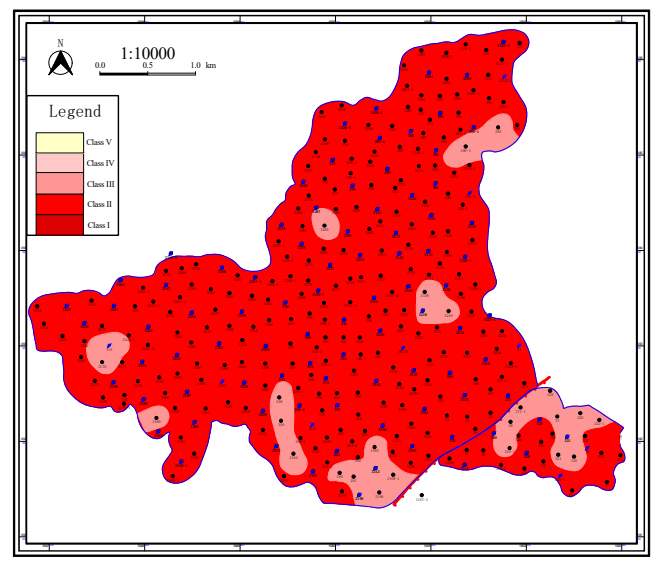

Fig.14 Distribution map of reservoir classification results in Chang 2 reservoir

Table 3. Evaluation criteria for NMR mobile fluid saturation

\begin{tabular}{cc}
\hline Mobile fluid saturation $/ \%$ & Reservoir classification \\
\hline$>65$ & Class I (good) \\
$50 \sim 65$ & Class II (better) \\
$35 \sim 50$ & Class III (middle) \\
$20 \sim 35$ & Class IV (poor) \\
$<20$ & Class V (very poor) \\
\hline
\end{tabular}

\subsection{Evaluation of oilfield development effect}

(technical)Recoverable reserves refer to the amount of oil and gas that can finally be produced from the oil and gas 
geological reserves under the given technical conditions. The recoverable reserves are not only related to the technology used in oilfield development, but also affected by well pattern and human factors in development. Therefore, the recoverable reserves predicted by dynamic method can indicate the development effect of the oilfield.

Movable reserves represent the recoverable degree of geological reserves, due to the heterogeneity of reservoir and fluid properties, the different recoverable degree of geological reserves in different parts of the reservoir and in different sections of the oil well, and the additional resistance existing in the oil-water two phase flow, in fact, a part of the movable reserves can not be produced. Therefore, the movable reserves are the limit value of the recoverable reserves. The closer the two, the better the oilfield development effect is. According to the classification standard of recovery factor in the oil and gas industry standard of the People's Republic of China (SY / T5838-93), and the characteristics of low permeability reservoirs, the development effects of oil fields are classified into 4 categories by the ratio of recoverable reserves to movable reserves (Table 4).
Table 4. Criteria for evaluating oilfield development effect by ratio of recoverable reserves to movable reserves

\begin{tabular}{ccccc}
\hline Target & Good & Better & Medium & Bad \\
\hline $\begin{array}{c}\text { Recoverable } \\
\text { reserves } /\end{array}$ & & & & \\
$\begin{array}{c}\text { movable } \\
\text { reserves } / \%\end{array}$ & $>85$ & $65-85$ & $45-65$ & $<45$ \\
\hline
\end{tabular}

The recoverable reserves of Chang 2 reservoir are predicted by water drive curve method, Tong Xianzhang's charter plate method and production decline method. According to Table 6, the recoverable reserves of Chang 2 reservoir are $410.5 \times 10^{4} \mathrm{t}($ table 5$)$, and the ratio of recoverable reserves to movable reserves is $64.2 \%$, according to the standard of Table 4, the actual development effect of the reservoir is medium to good, which shows that there is room for further adjustment in oilfield development.

Table 5. Recoverable reserves prediction results of Chang 2 reservoir performance method

\begin{tabular}{|c|c|c|c|c|}
\hline \multirow{2}{*}{ Method } & \multicolumn{3}{|c|}{ Recoverable reserves $/ 10^{4} \mathrm{t}$} & \multirow{2}{*}{$\begin{array}{l}\text { Recoverable reserves } \\
/ \text { movable reserves } / \%\end{array}$} \\
\hline & Faults south & Faults north & Total & \\
\hline water drive curve method & 12.96 & 396.52 & 409.5 & 64.1 \\
\hline $\begin{array}{l}\text { Tong Xianzhang's charter } \\
\text { plate method }\end{array}$ & 10.52 & 403.6 & 414.1 & 64.8 \\
\hline production decline method & $e^{13.65}$ & 394.18 & $\begin{array}{l}407.8 \\
410.5\end{array}$ & $\begin{array}{l}63.8 \\
642\end{array}$ \\
\hline
\end{tabular}

\section{Conclusions and recommendations}

(1) In Chang 2 reservoir, the pore radius is large, the micro-fine throat is developed, and the rock is dominated by small pore. Therefore, the movable fluid mainly exists in the small pore volume controlled by throat. The lower the permeability of rock, the more fluid exists in the volume controlled by throat, the lower the movable fluid saturation is, the more difficult the fluid is to be mined, and the correlation between the movable fluid saturation and the permeability is the strongest.

(2) The recoverable degree of Chang 2 reservoir reserves is $53.22 \%$, Class II and class III movable reserves are the main targets of oilfield development, and the total remaining movable reserves is $445.1 \times 10^{4} \mathrm{t}$, accounting for $69.7 \%$ of the movable reserves, which shows that there is great potential for adjustment in oilfield development. The adjustment is focused on the area corresponding to the remaining movable reserves of category III.

(3) The average movable fluid saturation of Chang 2 reservoir is $53.22 \%$, the reservoirs are mainly of type II (better) and type III reservoirs are relatively low, which shows that the reservoir development can achieve better results.

(4) The methods or ideas of oilfield development adjustment mainly include reperforating, increasing oil well production in main area and reducing oil well production in non-main area, intensifying water injection at the edge of reservoir and weakening water injection in the middle of reservoir, increasing viscosity of water injection.

(5) The conclusion of this paper is mainly based on laboratory experiment and reservoir engineering study, and its application effect needs to be verified by field practice.

\section{References}

1. Li Haibo. Core experiment study of NMR $T_{2}$ cutoff value[M]. Beijing: Chinese Academy of Sciences. 2008, 4-4.

2. Wang Weimin, Guo Heshen, Ye Zhaohui. The evaluation of development potential in low permeability oil field by the aid of NMR moveable fluid detecting technology[J]. ACTA 2001, 22(6): 4044.

3. Zhang Lunyou. Concepts of movable reserves and methods for determining economically recoverable reserves[J]. Natural Gas Exploration and Development, 1996, 19(4): 75-76. 
4. Liu Degnke, Sun Wwei, Ren Dazhong, et al. Features of pore-throat structures and movable fluid in tight gas reservoir: A case from the 8th member of Permian Xiashihezi Foramtion and the 1st member of Permian Shanxi Formation in the western area of Sulige Gasfield, Ordos Basin[J].Natural Gas Geoscience, 2016, 27(12): 2136-2146

5. Ren Dazhong, Sun Wei, Dong Fengjuan, et al. Characteristics of movable fluids in the Chang 81reservoir, Yanchang Formation in Huaqing oilfield, Ordos basin and the influencing factors[J]. Geology and Exploration, 2015, 51(4): 797-804.

6. Wang Ruifei, Chen Mingqiang. Characteristics and influencing factors of movable fluid in ultra-low permeability sandstone reservoir[J]. Acta Geologica Sinica, 2008, 29(4): 558-561.

7. Legchenko A, Beauce A, Baltassat J M, et al. Nuclear magnetic resonance as a geophysical tool for hydrogeologists $[\mathrm{J}]$. Journal of Applied Geophysics, 2002, 50(1): 21-46.

8. Cowan B. Nuclear magnetic resonance and relaxation[J]. Journal of Chemical Physics, 2010, 45(5): 185-187

9. Shi Diaodiao, Sun Sei, He Shengping. Relationship between micro-pore structure and movable fluid saturation in low permeability reservoir[J]. Geological Science and Technology Information, 2012, 31(4): 81-85

10. Huo Yingdong. Application of nuclear magnetic resonance technique in fluid saturation analysis of tight oil reservoir $[\mathrm{J}]$. West-china Exploration Engineering, 2018(6): 107-112.

11. Zhu Weiyao, Sun Yukai, Wang Shihu, et al. The filtration theory and method of effective development in ultra-low permeability reservoir. Beijing: Petroleum Industry Press, 2010: 31-35.

12. Yang Zhengming. Porous mechanisms of low permeability reservoirs and its application[D]. Beijing: Chinese Academy of Sciences, 2004, 73-80.

13. Cui Lianxun. Application of constant-rate intruding mercury and nuclear magnetic resonance method to low permeability reservoir evaluation[J]. Journal of Cheng Du of Technology (Science \& Technology Edition), 2012, 39(4): 430-433.

14. Wang Weimin, Guo Heshen, Ye Zhaohui. The evaluation of development potential in low permeability oilfield by the aid of NMR movable fluid detecting technology[J]. Acta Petrolei Sinica, 2001,22(6): 40-44

15. Ren Ying, Sun Wei, Zhang Xi, et al. Characteristics of movable fluids and study of production performance in different flow units of low - permeability reservoirs: An example from the Chang 6 block of the Jiyuan oilfield in Ordos Basin[J]. Geology and Exploration, 2016, 52(5): 974-984.

16. Hao Mingqiang, Li Shutie, Yang Zhengming, et al. The Effect of Relative Volume Percentage of Movable Fluid on Development Effects of Low
Permeability Reservoir[J]. Xinjiang Petroleum Geology, 2006, 27(3): 335-337.

17. Cao Baoge, Li Yuanliu, Xiao Ling, et al. Study on micropore structure characteristics of low permeability reservoir — Taking Chang 2 reservoir in Bailangcheng oil region as an example[J]. Xinjiang geology, 2019, 37(4): 536-539

18. Guo Ruiliang, Chen Xiaodong, Ma Miaofeng, et al. Analysis of the characteristics and its influencing factors of horizontal movable fluid in the Chang 7 tight reservoir in Longdong area, Ordos Basin[J]. Natural Gas Geoscience, 2018, 29(5): 665-673.

19. Ren Dazhong, Sun Wei, Lu Tao, et al. Microscopic Geological Factors of Movable Fluid Distribution in the Tight Sandstone Gas Reservoir: Taking the He 8 Reservoir in the East of Sulige Gas Field as An Example[J]. Geoscience, 2015,29(6): 1409-1417.

20. He Gengsheng, Tang Hai. Reservoir physics. Beijing: Petroleum Industry Press, 2011: 48-48.

21. Zheng Rui, Liu Linyu, Li Nanxing. Study on the fourproperty relationship and the lower limit of effective thickness of Chang 2 reservoir in Bailangcheng Area[J]. Petroleum Geology and Engineering, 2011, 25(2): $33-35$

22. Song Ziqi, Cheng Guojian, Wang Jing, etal. Determination of effective thickness for oil reservoirs with extra-low permeability[J]. Acta Petrolei Sinica, 2006, 27(6): 103-106.

23. Wu Xiaobo, Wang Shixing, Xiao Chunsheng. A new study of Delaunay triangulation creation[J]. Acta Geodaetica et Cartographica Cinica, 1999, 28(1): 2835.

24. Li Pan, Sun Wei1, Li Changzheng, et al. Characteristics of Movable Fluids in the Low Permeability Sandstone Reservoir: Taking the Chang 8 reservoir of Ma Ling Oilfield, Ordos Basin as An Example[J]. Progress in Geophysics, 2018: 1-10. 